Kasai, Masataka

[25]

\section{Acceleration of cell division independent of increases in cell mass} $\&$ Haruo Okado ${ }^{3}$

${ }^{1}$ National Institute of Infectious Diseases, Tokyo, Japan

${ }^{2}$ University of Tokyo, Tokyo, Japan

${ }^{3}$ Tokyo Metropolitan Institute for Neuroscience, Tokyo, Japan

Although most cells maintain a constant size as they proliferate, it remains unclear identified a DNA-binding protein, Translin, whose native form consists of a ringmeric structure creates a DNA-binding domain for the sequences at breakpoint gene on human chromosome 2q21 also harbors the ERCC3 gene, which is known to be involved in human disorders of DNA repair. Despite detailed studies on protein-DNA interactions, the functional significance of Translin remains unknown. In studies comparing basal expression levels of Translin across various tissues and cell lines, we have found a good correlation between Translin protein synthesis and cell proliferation. To analyze the effect of Translin on cell proliferation, we established a Translin expression system regulatable by doxycycline. We found that overexpression of Translin in HEK293 cells increased cell proliferation by two- to threefold. Flowcytometric forward-scatter analysis revealed that the size of the cells was increased compared with the control cells that grew in the absence of doxycycline. These results indicate that Translin may be involved in cell cycle progression and that its overexpression accelerates cell division independent of increases in cell mass.

Khan, Javed

[26]
Masataka Kasai ${ }^{1}$, Hiroko Sato ${ }^{1}$, Reiko Ishida ${ }^{1}$, Katsunori Aoki ${ }^{2}$ how the cell cycle progression is coupled to increases in cell mass. We have previously shaped structure with an assembly of eight $27-\mathrm{kD}$ monomer subunits. This multijunctions in many chromosomal translocations. The region around the Translin

\section{Development of a molecular taxonomy of small blue round-cell tumors using cDNA microarrays}

Javed Khan ${ }^{1}$, Jun Wei ${ }^{1}$, Lao Saal ${ }^{1}$, Ladanyi Marc ${ }^{2}$, Ringnér Markus ${ }^{3}$, Carsten Peterson ${ }^{3}$, Yidong Chen ${ }^{1}$ $\&$ Paul Meltzer ${ }^{1}$

${ }^{1}$ Cancer Genetics Branch, National Human Genome Research Institute, National Institutes of Health, Bethesda, Maryland, USA

${ }^{2}$ Memorial Sloan-Kettering Cancer Center, New York, New York, USA

${ }^{3}$ Department of Theoretical Physics, Lund University, Lund, Sweden

Many of the pediatric solid tumors categorized as small blue round-cell tumors (neuroblastoma, rhabdomyosarcoma, lymphoma and Ewing sarcoma) are difficult to distinguish morphologically, and routine immunohistochemistry may be insufficient to characterize them. Several specialized techniques are used to diagnose these cancers, including cytogenetics, interphase fluorescence in situ hybridization, the polymerase chain reaction with reverse transcription, and immunohistochemistry. However, despite the availability of these tests, poorly differentiated cancers can still pose a diagnostic dilemma. Gene expression profiling permits the simultaneous analysis of multiple markers, no one of which can perfectly classify any cancer. Based on our hypothesis that the gene expression profile is specific for each type, we have used complementary DNA microarrays containing 6,570 genes to investigate the expression profiles of 64 pediatric cancers. These included both tumor biopsy tissues (13 Ewing sarcomas and 11 rhabdomyosarcomas) and cell lines (10 Ewing sarcomas, 10 rhabdomyosarcomas, 12 neuroblastomas and 8 lymphomas). Using supervised clustering ( $F$-statistics and weighted gene analysis), multidimensional scaling, hierarchical clustering, principal component analysis and layered perceptron prediction algorithms, we have identified 131 genes that accurately distinguish between these cancers. We have confirmed the accuracy of these findings in classification of these cancers on 12 blind samples. We have also identified several biologically relevant genes that are uniquely expressed in specific cancer types but that have not been previously associated with these diseases. Our results support the potential of cDNA microarrays as an efficient tool for developing a molecular taxonomy of cancer.

Kinoshita, Ichiro

[27]

\section{Identification of genes regulated by AP-1 in Rat-1a cells expressing inducible cJun}

Ichiro Kinoshita ${ }^{1}$, Virna Leaner ${ }^{1}$, Motoo Katabami ${ }^{1}$, Denver Hendricks ${ }^{1}$, Rammon Manzano ${ }^{1}$, Aaron Bell ${ }^{1}$, Paul Dent ${ }^{2} \&$ Michael Birrer ${ }^{1}$

${ }^{1}$ Cell and Cancer Biology Department, National Cancer Institute, 9610 Medical Center Drive, Rockville, Maryland 20850, USA

${ }^{2}$ Department of Radiation Oncology, Massey Cancer Center, Medical College of Virginia, Virginia Commonwealth University, Richmond, Virginia 23298, USA

A major component of the transcription complex AP-1 is cJun. Although upstream signaling cascades to this transcription factor have been well characterized, its downstream effector genes remain to be characterized. To identify cJun target genes, we established a conditional transformation system of Rat-1a cells using tetracycline-inducible cJun. Under adherent growth conditions, cJun expression was rapidly increased in $4 \mathrm{~h}$, and morphological changes gradually occurred after $24 \mathrm{~h}$ (spindle and refractile phenotypes appeared after $24 \mathrm{~h}$; close contact of cells, after 2 days; and piling up of cells, after 3 days). In addition, expression of cJun allowed Rat-1a cells to grow without a substratum (nonadherent growth). We identified differentially expressed genes from cJun-induced cells compared with uninduced Rat-1a cells using differential display, suppression subtractive hybridization and microarray analysis. We identified 27 upregulated and 8 downregulated cDNA fragment clones and confirmed 2- to 28-fold differences by northern blot analysis. Sequence analysis revealed 29 known genes and five unmatched clones in addition to $c$-jun itself. All 11 genes with known promoters had AP-1 sites, and 5 genes have been reported to be regulated by AP-1. Surprisingly, approximately $50 \%$ of the genes were identical to or showed homology with cytoskeleton- and adhesion-related genes, suggesting possible cooperative roles in morphological transformation. Furthermore, growth under nonadherent conditions altered the differential expression of some genes. We are currently investigating the precise roles of these genes.

Kirsch, Ilan

[28]

\section{The Cancer Chromosome Aberration Project: Update on BAC clone repository} and database

Ilan Kirsch ${ }^{1}$, Raluca Yonescu ${ }^{1}$, Theresa Brown ${ }^{1}$, Tricia Reppert $^{1}$, Turid Knutsen ${ }^{1}$, Karl Sirotkin ${ }^{2} \&$ Thomas Ried $^{1}$

${ }^{1}$ Genetics Department, Medicine Branch, Division of Clinical Sciences, National Cancer Institute, National Institutes of Health, Bethesda, Maryland, USA ${ }^{2}$ National Center for Biotechnology Information, National Institutes of Health, Bethesda, Maryland, USA

The Cancer Chromosome Aberration Project generates a repository of BAC clones with a resolution of 1-2 megabases. The cytogenetic location of all clones is established by high-resolution fluoresence in situ hybridization mapping onto 
Abstracts: Session II

prometaphase chromosomes. Linkage to existing physical maps is provided by the requisite presence of a mapped sequence tagged site in all selected BACs and in many cases by the full or end sequencing of the mapped clone itself. Clones in the repository should allow all chromosomal breakpoints to be localized to within 2 $\mathrm{Mb}$ and provide entry points to existing physical maps of the intervening distance. With the completion of a rough draft of the human genome, sequences between mapped breakpoints can be immediately queried for potential candidate genes. We will provide an update on the number of BAC clones and chromosomes currently mapped and available to the biomedical community. One critical issue is the integration of databases for the cytogenetic and physical maps. The National Cancer Institute and National Center for Biotechnology Information are working together to develop a meaningful integration that provides (1) a direct connection between catalogued and newly discovered chromosomal breakpoints or regions of genomic imbalances and the BAC clone set; (2) direct display of recurring chromosomal breakpoints that coincide with BAC locations; (3) placement of mapped BACs on the human sequence; (4) tools to build contiguous clone sets; (5) integration of the BAC clone set with databases for comparative genomic hybridization and spectral karyotyping and (6) an interface for the identification of orthologous regions of chromosomal aberrations in human cancers and their respective mouse models.

Klein, Christoph [29]

\section{Transcriptome and genome analysis of single disseminated tumor cells: approach to study minimal residual cancer}

Christoph Klein ${ }^{1}$, Stefan Seidl ${ }^{1}$, Karina Petat-Dutter ${ }^{1}$, Oleg Schmidt-Kittler ${ }^{1}$, Sonja Offner ${ }^{2} \&$ Gert Riethmüller $^{1}$

${ }^{1}$ Institut für Immunologie, Ludwig-Maximilians-Universität, Munich, Germany ${ }^{2}$ Micromet GmbH, Martinsried, Germany

Despite increasing molecular-genetic understanding of the development of malignant epithelial neoplasias, the front-line therapy for patients with carcinomas is still surgery. Because systemic adjuvant treatments such as chemotherapy or immunotherapy have had limited success and because the characteristics of systemically disseminated tumor cells can be very different from those of the primary tumor or end-stage metastasis, we have studied the evolution and progression of systemic cancer directly. We have developed techniques to detect, isolate and study genomes and transcriptomes of single micrometastatic cells present in bone marrow of carcinoma patients. The first results of our genetic analysis demonstrate that disseminated tumor cells are clonally related, indicating their selection from heterogeneous cell populations of the primary tumor. Gene expression analysis of single disseminated tumor cells revealed their engagement in specific functional activities, such as tissue invasion, proliferation and DNA damage repair. Detailed analyses of single disseminated cells will help determine which genotypes and phenotypes are selected during dissemination, which cells survive in the new environment and what governs the establishment of a metastasis. This knowledge will be useful in selectively targeting the precursor cells with adjuvant therapies long before metastatic disease is clinically evident and incurable.
Krahe, Ralf

$[30]$

\section{Gene expression profiling reveals fundamental biological differences in AML with trisomy 8 and normal cytogenetics}

Kimmo I. Virtaneva ${ }^{1}$, Fred A. Wright ${ }^{1}$, Stephan M. Tanner ${ }^{1}$, Bo Yuan ${ }^{1}$, William J. Lemon ${ }^{1}$, Michael A. Caligiuri2,3, Clara D. Bloomfield ${ }^{2,3}$, Albert de la Chapelle ${ }^{1} \&$ Ralf Krahe $^{1}$

${ }^{1}$ Division of Human Cancer Genetics, Department of Molecular Virology, Immunology and Medical Genetics, Comprehensive Cancer Center, Ohio State University, Columbus, Ohio 43210, USA

${ }^{2}$ Division of Hematology/Oncology, Department of Internal Medicine, Comprehensive Cancer Center, Ohio State University, Columbus, Ohio 43210, USA ${ }^{3}$ Cancer and Leukemia Group B, Chicago, Illinois 60604, USA

Acute myeloid leukemia (AML) is a heterogeneous group of diseases. Patients exhibiting normal cytogenetics (AML-CN) constitute the single largest group, and trisomy $8(\mathrm{AML}+8)$ as the sole abnormality is the most frequent trisomy. How trisomy contributes to tumorigenesis is unknown. Because hematopoietic differentiation is predominantly regulated at the transcriptional level, we proposed that, whatever the underlying molecular leukemogenic event(s) associated with AML$\mathrm{CN}$ and $\mathrm{AML}+8$, the molecular changes at the DNA level should be reflected in specific changes at the RNA level. We used oligonucleotide-based DNA microarrays to study global gene expression in ten $\mathrm{AML}+8$ patients with +8 as the sole chromosomal abnormality and ten AML-CN patients, as well as seven CD34+ cell samples purified from normal bone marrow of healthy individuals as a representative heterogeneous population of stem and progenitor cells. For the 6,606 genes studied, expression patterns of AML patients were clearly distinct from those of CD34+ cells of normal individuals. AML +8 was associated with an overexpression of genes on chromosome 8 , suggesting a role for gene dosage effects in the etiology of AML +8 . Systematic analysis by cellular function indicated upregulation of genes involved in cell adhesion in both AML groups. We observed other highly significant results in the comparison of $\mathrm{AML}+8$ with AML-CN for genes involved in the regulation of apoptosis, suggesting a fundamental biological difference in programmed cell death. The observed differences in the dysregulation of specific functional subsets of genes between the two AML subclasses merit further functional studies of the individual components of the pathways.

Krupke, Debra M.

[31]

\section{Electronic access to data from mouse cancer models: The Mouse Tumor Biology database}

Debra M. Krupke, Carol J. Bult, Dieter Naf, John P. Sundberg \& Janan T. Eppig

Jackson Laboratory, 600 Main Street, Bar Harbor, Maine 04609, USA

The Mouse Tumor Biology database (MTB) has been developed to provide electronic access to mouse tumor biology data. The primary focus of MTB is spontaneous and induced tumors in genetically defined mice (inbreds, hybrids, mutants and genetically engineered strains). By making such data readily available online, MTB provides the scientific community with a much-needed, easily accessible central resource for rapidly finding and evaluating the expanding volume of mouse tumor data. MTB provides cancer researchers with access to data on mouse models for cancer and includes information such as tumor names and classifications, pathology reports, histopathological images, genetics of the strain, genomic changes in the tumor, strain names, tumor frequency and latency, references and 